\title{
Investigation of the ion permeability of poly(p-xylylene) films
}

\author{
Phillip Hanefeld, Frank Sittner, Wolgang Ensinger, Andreas Greiner*
}

Philipps-University Marburg, Department of Chemistry, Hans-Meerwein-Str., D-35032 Marburg, Germany, e-mail: greiner@staff.uni-marburg.de

(Received: 25 July, 2005; published: 02 June, 2006)

\begin{abstract}
The ion permeability of poly(p-xylylene) (PPX, parylene- $\left.N^{\circledR}\right)$ thin films was investigated using a cyclovoltammetric setup. Iron rods of different surface roughness were coated with PPX films of different thickness $(140-700 \mathrm{~nm})$ by vapour phase deposition (Gorham process). The presence of pinholes in PPX films deposited as described was analyzed by ion permeability according to cyclovoltammetric measurements. Current densities were directly related to the area of iron not covered by the polymer. It was found that films deposited on iron surfaces by the Gorham process showed significant ion permeability for film thicknesses below $500 \mathrm{~nm}$ and no permeability at all for films equal to or thicker than $700 \mathrm{~nm}$, which indicated the absence of pinholes of PPX films of $700 \mathrm{~nm}$ thickness. Furthermore, films of $140 \mathrm{~nm}$ were deposited on iron surfaces of different roughness (the mean roughness was measured to be $10^{2} \mathrm{~nm}, 10^{3} \mathrm{~nm}$ and $10^{5} \mathrm{~nm}$ ). Current density increased by the increase in roughness, which confirmed that pinhole formation correlated with surface roughness.
\end{abstract}

\section{Introduction}

Poly(p-xylylene) (PPX) prepared by the so-called Gorham process via vapour phase deposition of quinodimethane is considered to be a conformal coating process [1]. Its excellent wear resistance, chemical inertness, high thermal stability, transparency, biocompatibility, and dielectric properties makes PPX an ideal coating material for a variety of applications including sensors, medical implants, and microelectronics just to name a few [2]. Recently, vapour deposition of PPX has been used for the preparation of PPX nanotubes by the TUFT-process (tubes by fibre templates) [3]. Following the Gorham-process, conformal coatings of PPX are obtained by vaporization, and pyrolyzation of [2.2]paracyclophane and subsequent deposition on nearly any solid substrate (Fig. 1). The spontaneous polymerization of pquinodimethane at reduced pressure takes place below $30^{\circ} \mathrm{C}$. The Gorham process is of particular interest for a variety of high-end applications since neither solvents nor catalyst are required and no side-products are usually observed. However, the application of PPX films as protective coatings requires pinhole free films, which may depend on factors such as film thickness, deposition conditions, and the nature of the substrate.

Electrochemical measurements using cyclovoltammetry have been used successfully before for evaluation of pinholes of carbon-coatings [4,5]. Goal of this contribution is the introduction of a new method for the evaluation of pinholes in Gorham-type PPX films applying electrochemical measurements. 


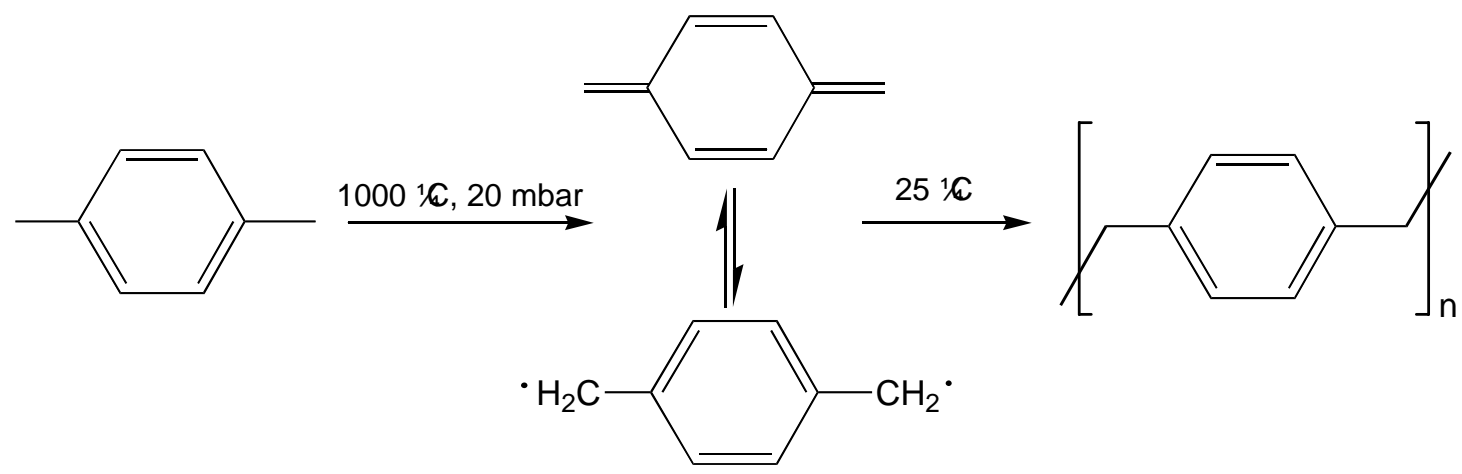

Fig. 1. Schematic view of the PPX synthesis using the CVD method.

\section{Results and Discussion}

Information about the dissolving process can be extracted from the shape of the current density versus potential of the dissolving metal ions, taking place on the sample's surface by flow of the dissolved ions through pinholes present in the polymer coating [6]. Fig. 2 shows a plot for an iron sample, coated with a PPX film $140 \mathrm{~nm}$ of thickness. At large negative potentials hydrogen is generated at the metal surface and the iron electrode acts as cathode. At the so-called open circuit potential $E_{\text {corr. }}$ current densities of cathodic and anodic reactions become equal, therefore the resulting measured current density becomes zero. From this point, with increasing anodic potential the iron electrode starts to dissolve, i.e. it corrodes with increasing current density until a maximum density is reached. Here, the so-called critical current density $I_{\text {crit. }}$ is reached and a passivation takes place. The iron surface is covered by iron oxide and iron hydroxide, leading to a reduction in dissolution current until it is no longer dependent on the applied potential.

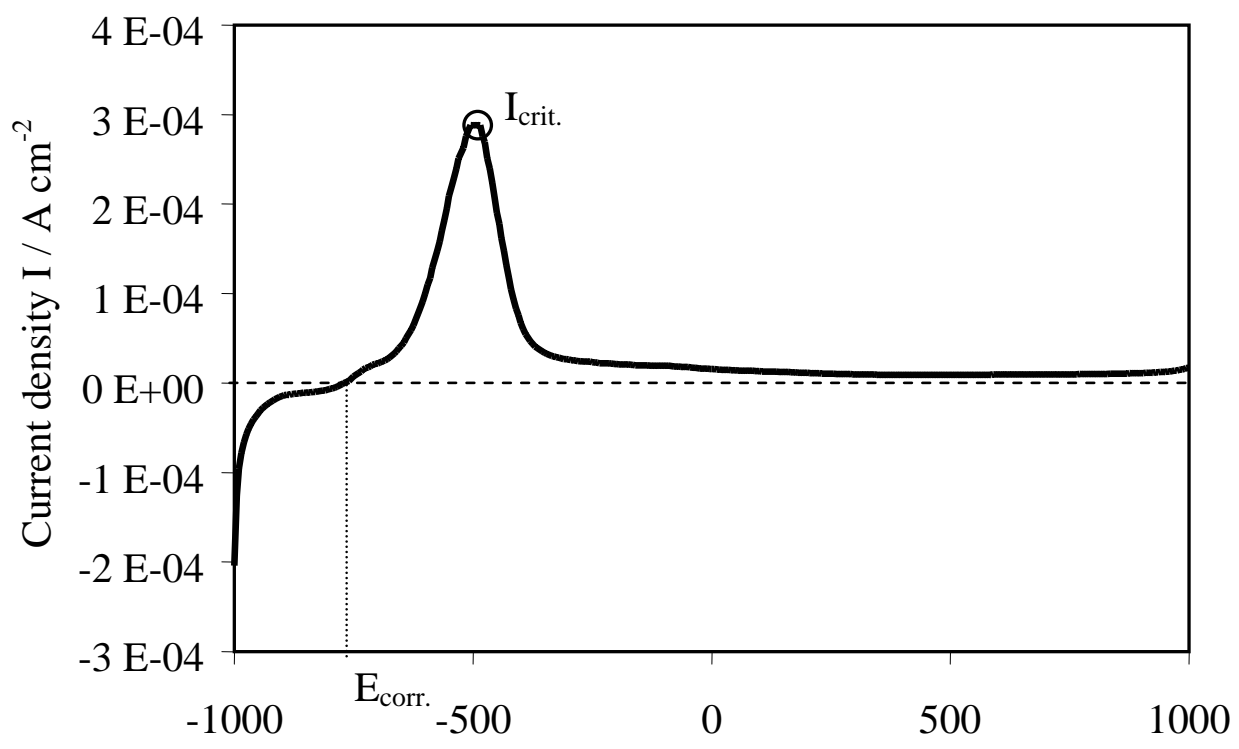

Potential E / mV

Fig. 2. Current density vs. potential plot of a mirror-polished iron sample coated with $140 \mathrm{~nm}$ PPX.

Since the maximum current density as well as the final passivation current density are both proportional to the rate of corrosion of the metal surface, it is possible to 
compare the $I_{\text {crit.. }}$ values of different samples to obtain information about their corrosion behaviour. Since the PPX coating material is absolutely insulating, iron dissolution can only take place, where the iron substrate is in contact with the aqueous medium: at flaws and pinholes in the coating layer. Therefore, the dissolution current is proportional to the ion permeability of the coating polymer. The higher the measured current density is, the higher the coating's porosity must be and the higher is the quantity and the size of the pinholes which can be found in the protective layer.

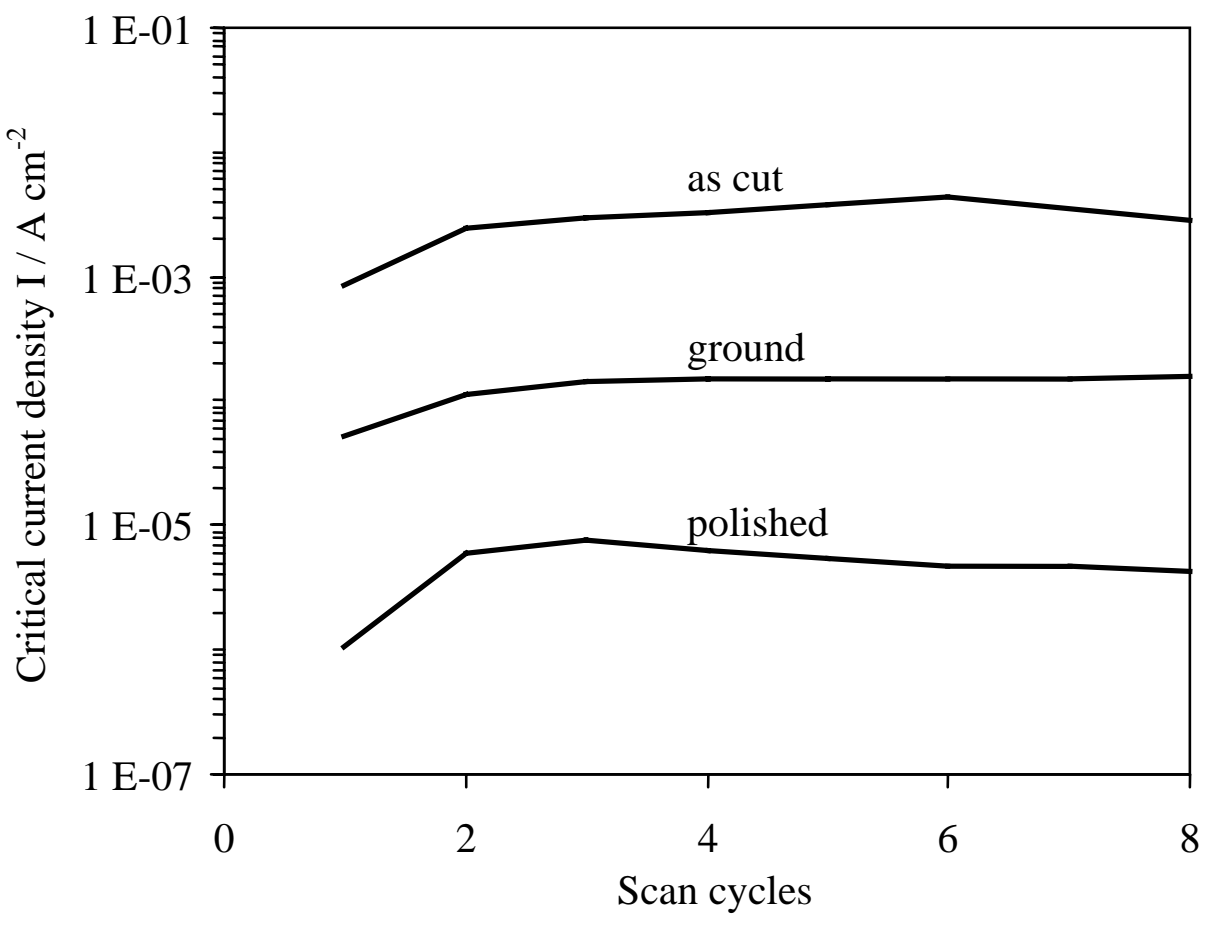

Fig. 3. The critical current densities vs. number of scan cycles; with increasing surface roughness dissolution currents increase as well.

Fig. 3 shows plots of the critical current density versus the number of scan cycles for samples with differently treated iron surfaces. The mean roughness of these iron samples was determined using a surface-profilometer and was found to be $10^{5} \mathrm{~nm}$ for the cut, $10^{3} \mathrm{~nm}$ for the grinded and $10^{2} \mathrm{~nm}$ for the polished sample (Fig. 4). As can be seen, the highest current densities are found for the untreated or roughest sample, which means it has the highest porosity. The ground sample, where the polishing step has been left out, shows a lower current density. The polished sample with the smoothest surface shows the lowest dissolution current. That means that a bigger surface roughness leads to coatings with more or bigger pinholes. A homogeneous spreading of the gas molecules over the sample during the coating process due to the small mean free path may be found in the case of macroscopic edges but not on a microscopic scale.

As a consequence different surfaces may require different film-thicknesses to guarantee a real pinhole-free, uniform coating. For polished iron samples the results are shown in Fig. 6. Samples with increasing film-thicknesses have been investigated and iron dissolution current densities could be measured up to $700 \mathrm{~nm}$. Above this value the typical curve-shape disappears and only noise is measured, which means that above $700 \mathrm{~nm}$ the coatings are pinhole-free since there is no contact of the metal substrate to the aqueous electrolyte. 


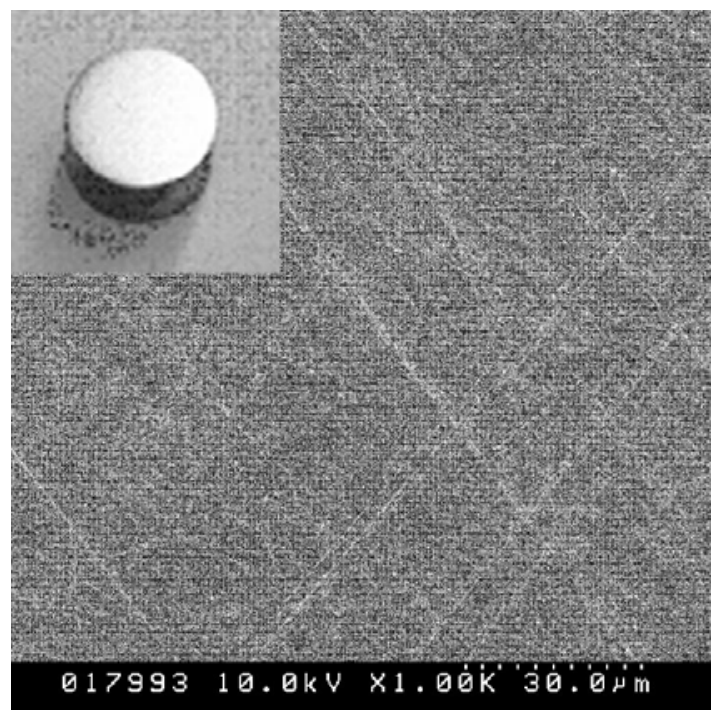

(a)

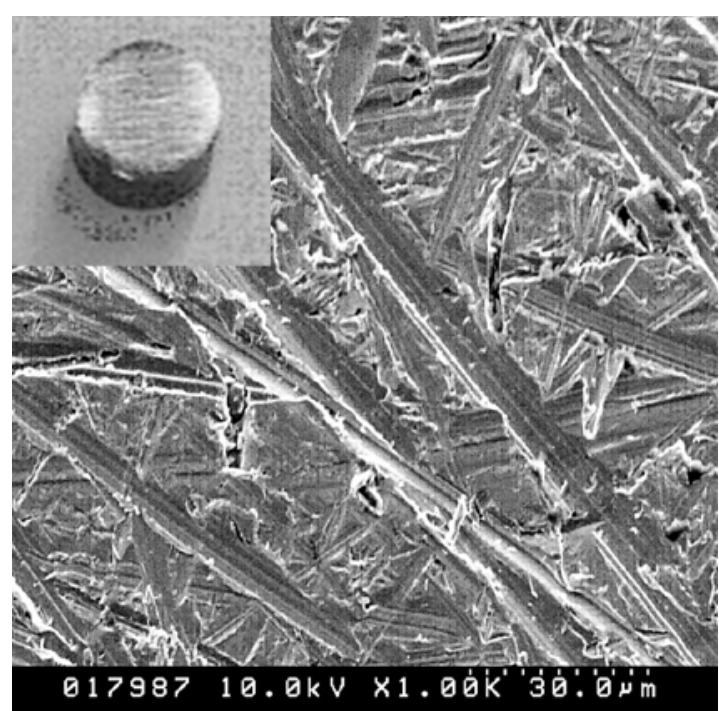

(b)

Fig. 4. Macroscopic (insets) and microscopoic (scanning electron microscope) images of surfaces of mirror-polished (a) and ground (b) iron samples

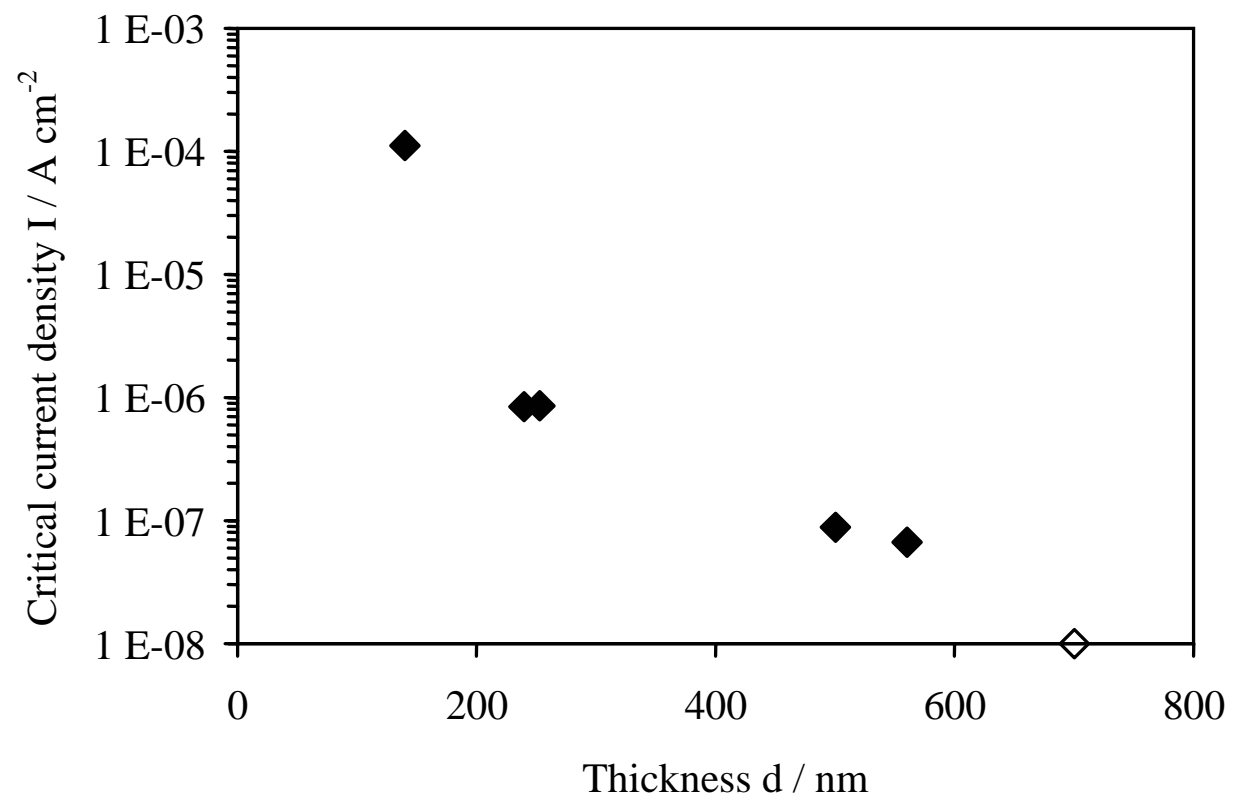

Fig. 5. Dissolution current densities vs. increasing PPX-film thickness on iron samples.

\section{Conclusions}

Cyclic voltammetry measurements show that PPX coatings deposited by the Gorham-process do not necessarily lead to uniform and pinhole-free films. A minimum thickness has to be reached depending on the substrate. This has also been predicted by theoretical models for the deposition of CVD polymers in literature. The value of this thickness is strongly dependent on the sample's topography and chemical composition. For different metals, Vaeth et al. found that deactivation of the reactive precursor $\mathrm{p}$-chinodimethane can take place due to interactions with the chemically active surface. $[7,8]$ In this case, polymerization on the surface of these metals (e. g. $\mathrm{Cu}, \mathrm{Ag}, \mathrm{Fe}$ ) is inhibited until a first layer of organic deposits is formed. Afterwards, polymerization is no further affected by the nature of the underlying material. The thickness of these deactivated layers was reported to be several $\mathrm{nm}$, 
indicating that the chemical composition of the surface plays no dominant role in our study. Regarding the topological aspect of the surface, macroscopic small edges and holes may be covered by the polymer but microscopic roughness can still be the origin for pinholes and leaks in the deposited film. By depositing films of different thickness on equivalently rough iron samples, we have found the minimum thickness for pinhole-free layers of PPX on mirror-polished iron to be around $700 \mathrm{~nm}$. Since similar results have been found by other techniques in the past, cyclovoltammetry is suitable as a technique to determine the presence and quantity of pores in polymer films in general.

\section{Experimental part}

\section{Sample preparation}

To measure the polymer porosity, films were deposited on iron cylinders with $1 \mathrm{~cm}$ height and a diameter of $0.9 \mathrm{~cm}$, cut from an iron (99.8\%) rod. The cylinder's flat surface was treated to a mirror-like finish in a grinding and polishing process. In a final step, a paste of natural diamonds of $1 \mu \mathrm{m}$ size was used to ensure constant surface properties of the iron samples. For some samples the final step was left out, leaving a rough surface, treated only with grinding paper (grain: 600). Some of the samples remained untreated after having been cut from the rod as reference samples. This was done to investigate the influence of the surface roughness on the film porosity.

\section{Polymer deposition}

Each sample, together with a smooth glass plate as reference for thickness measurements, was placed inside the deposition chamber, which was evacuated until a pressure of $2 \mathrm{kPa}$ was reached. The coating process consisted of three steps, carried out in a SCS Labcoater, model 2010. First, 100 to $500 \mathrm{mg}$ of [2.2]paracyclophane were vaporized at about $175^{\circ} \mathrm{C}$ at a pressure of $2 \mathrm{kPa}$ from an aluminium crucible. [2.2]paracyclophane was pyrolyzed at $650^{\circ} \mathrm{C}$ forming $1,4-$ quinodimethane. After entering the deposition chamber 1,4-quinodimethane condensed on the substrate's surface at around $35^{\circ} \mathrm{C}$ with spontaneous polymerization, forming a PPX film covalently bound to the metal surface (Fig. 1).

\section{Cyclovoltammetric measurements}

The polymer-coated iron cylinders were electrically contacted with a conductive silver-glue at the backside and then covered with an insulating varnish, leaving only the frontal coated surface uncovered. The electrode prepared in this way was integrated into a cyclovoltammetric measuring system with a three-electrode-setup, including the sample electrode, a graphite counter-electrode and a saturated calomel reference-electrode (SCE) [6]. The electrolyte medium was an acetate buffer of $\mathrm{pH}$ 6.75 at $20{ }^{\circ} \mathrm{C}$. Measurements and data processing were carried out with a potentiostat from EG\&G (model 273A) controlled by the software Soft Corr III. Cyclic voltammetry was used in the potential range between $-1 \vee$ and $+1 \vee$ vs. SCE with a potential scanning speed of $10 \mathrm{mV} \mathrm{s}^{-1}$. The current density of iron dissolution through the micropores in the coating (Fig. 6) was extracted from the current potential plots of the forward scan and plotted against the number of scan cycles. 


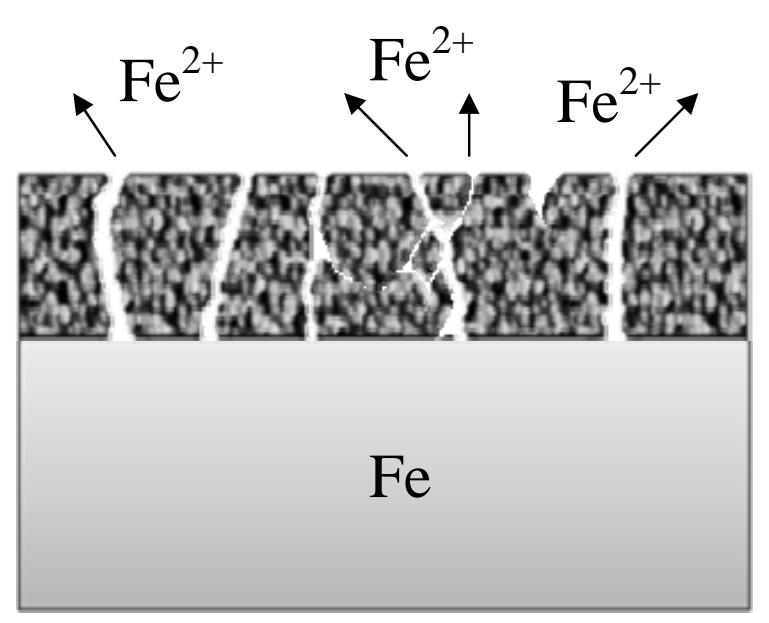

Fig. 6. Schematic view of a porous PPX film on an iron substrate. The dissolution current of the $\mathrm{Fe}^{2+}$-ions flow through the micropores is measured.

\section{Surface roughness and film thickness determination}

The resulting film thickness of every sample was determined by cutting the polymer film deposited on the reference glass plate. The difference in height between the glass and the film at the edge of the cut was measured using a Dektak ${ }^{\circledR}$-profilometer. For each film, three measurements were done and the mean value was used for discussions.

The surface roughness of the reference steel rods was measured by using the same method. The surface structure was recorded at five spots on the cylinder and the mean deviation of the peaks and valleys from the median was calculated and used as the roughness of each sample.

\section{Acknowledgements}

We would like to thank Specialty Coating Systems, Indianapolis, U.S.A., for the kind support and donation of parylene monomer.

\section{References}

[1] (a) Beach, W. F.; Lee, C.; Bassett, D. R.; Austin, T. M.; Olson, R. in: Encyclopedia of Polymer Science and Technology, 1989, pp. 990-1025, Wiley \& Sons. (b) Greiner, A.; Mang, S.; Schäfer, S.; Simon, P.; Acta Polym. 1997, 48, 1.

[2] Gorham, W. F.; J. Polym. Sci. A-1 1966, 4, 3027.

[3] (a) Bognitzki, M.; Hou, H.; Ishaque, M.; Frese, T.; Hellwig, M.; Schwarte, C.; Schaper, A.; Wendorff, J. H.; Greiner, A.; Adv. Mater. 2000, 12, 637. (b) Caruso, R.; Schattka, J. H.; Greiner, A.; Adv. Mater. 2001, 13, 1577. (c) Hou, H.; Zeng, J.; Schaper, A.; Wendorff, J. H.; Greiner, A.; Macromolecules 2002, 35, 2429.

[4] Sittner, F; Enders, B; Jungclas, H.; Ensinger, W., Surf. Coat. Tech. 2002, 158$159,368$.

[5] Heintz, E. L. H.; Kranz, C.; Mizaikoff, B.; Noh, H.-S.; Hesketch, P.; Lugstein, A.; Bertagnolli, E.; IEEE-NANO 2001, Maui, Hawaii, USA, 28-30 October 2001.

[6] Ensinger, W.: "Protection against aqueous corrosion by ion implantation and ion beam assisted deposition", in: Pauleau, Y.; Barna, P. B. (eds.); "Protective Coatings and Thin Films: Synthesis, Characterization and Applications", Kluwer Academic Publishers, Dordrecht, 1997, p. 585.

[7] Vaeth, K. M.; Jensen, K. F.; Chem. Mater. 2000, 12, 1305.

[8] Vaeth, K. M.; Jackman, R. J., Black, A. J.; Whitesides, G. M.; Jensen, K. F.; Langmuir 2000, 16, 8495. 